\title{
Arteriovenous malformation of the mandible: a life-threatening situation
}

\author{
Nikolaos Sakkas • Alexander Schramm • \\ Marc C. Metzger • Ansgar Berlis • \\ Rainer Schmelzeisen • Joerg-Elard Otten • \\ Bettina Hohlweg-Majert
}

\begin{abstract}
Arteriovenous malformation (AVM) of the mandible is not only a rare entity, but also one that can be potentially life threatening due to massive haemorrhage. The authors describe three cases of children with AVM of the mandible.
\end{abstract}

\section{Introduction}

Arteriovenous malformations (AVMs) of the mandible are rarely encountered in clinical practice. Nevertheless, it is essential that dentists, oral surgeons and radiologists are able to recognise these lesions because of their potentially life-threatening complication. They are opposed to an abnormal proliferation of endothelial cells of a haemangioma, occur as a result of errors in embryogen-

\author{
N. Sakkas • M. C. Metzger • R. Schmelzeisen - J.-E. Otten • \\ B. Hohlweg-Majert $(\bowtie)$ \\ Department of Oral and Maxillofacial Surgery, \\ University Hospital Freiburg, \\ Hugstetter Str. 55, \\ 79106 Freiburg, Germany \\ e-mail: bettina.majert@uniklinik-freiburg.de

\section{A. Schramm} \\ Department of Oral and Maxillofacial Surgery, \\ University Hospital Hannover, \\ Carl-Neuberg Str. 1, \\ 30625 Hannover, Germany \\ A. Berlis \\ Department of Neuroradiology, University Hospital Freiburg, \\ Hugstetter Str. 55, \\ 79106 Freiburg, Germany
}

esis, are always present at birth, but often go unrecognised for years [1].

Gold standard treatment of AVMs is an endovascular embolisation, combined with surgery $[2,3]$. Embolisation occludes the feeding vessels of the lesion. The surgical intervention in the maxillofacial region implies a partial or complete resection of the mandible. Repair of form and function may be challenging, especially in children.

\section{Materials and methods}

\section{Case 1}

A 14-year-old boy was presented to our department with recurring and excessive periodontal bleeding from the region of the left lower first molar (tooth 36 or 19) on the occasion of conservative dental treatment. The first suspected diagnosis of AVM was established in August 1997 by a panoramic radiograph on the occasion of periodontal bleeding of the left mandible. The diagnosis was then confirmed through an angiogram (Fig. 1a). Due to lifethreatening intraoral bleeding in November 1997 and March 1998, the boy was hospitalised. During hospitalisation, local haemostatic measures and blood transfusion were carried out. In April 1998, an arterial emergency embolisation of the AVM was performed due to massive haemorrhage using Ethibloc ${ }^{\circledR}$ (Ethicon, Johnson \& Johnson, Düsseldorf, Germany). The subsequent first surgical intervention (Fig. 1b) consisted of ligation of the left external carotid artery, the right facial artery (due to contralateral reperfusion of the AVM), excision of the soft tissue extension of the lesion and intraosseous application of fibrin glue. The next 18 months were uneventful, but due to relapsing and massive intraoral haemorrhage, the patient was once again 
hospitalised. The angiogram showed a reperfusion of the AVM from collateral arteries originating from the left occipital artery. Emergency intraosseous embolisation with butyl-2-cyanoacrylate (Histoacryl ${ }^{\circledR}$, Braun, Melsungen, Germany) was undertaken followed by elective embolisation through the left occipital artery and ligation of the vessel. Four weeks later, the intraosseous embolisation was repeated, and a postoperative CT scan was taken (Fig. 1c). The AVM was permanently obliterated, and no further periodontal bleeding occurred.

Approximately 1 year later (1999), the patient was presented with a spontaneous fracture of the left angle of the mandible (Fig. 1d). Necrotic mandibular bone and exposed embolisation material were visible intraorally. The

Fig. 1 a Angiogram showing an AVM in the body of the left mandible. b Massive intraoperative haemorrhage from the AVM. c Postoperative CT examination showing the filling of the cavity of the AVM with embolisation material. d Panoramic radiograph showing the spontaneous fracture of the mandible. e Panoramic radiograph after fibular flap reconstruction and removal of the plates
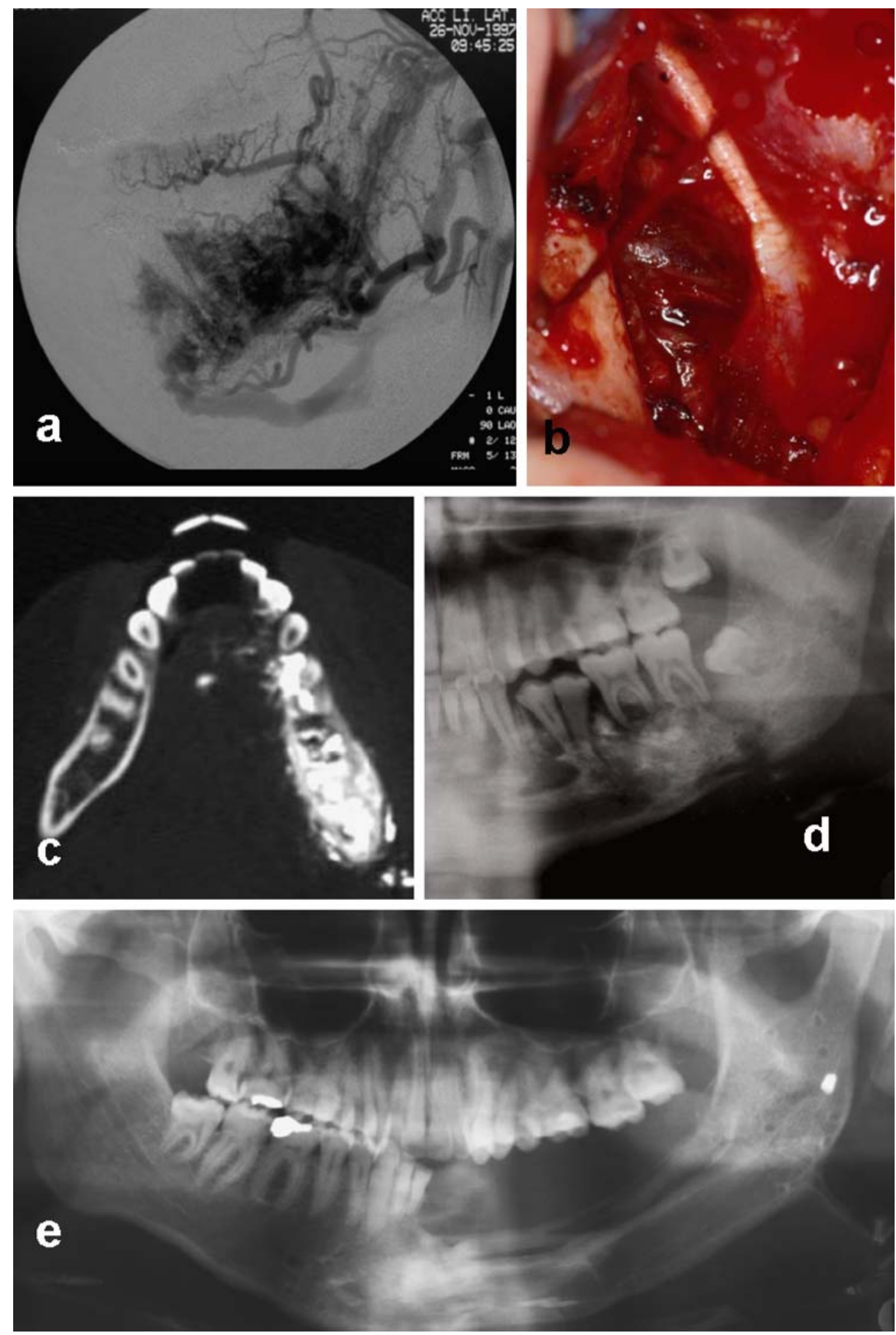
patient was treated conservatively over a period of 2 months, and the wound was irrigated frequently with antibacterial solution. Because the parents rejected a primary reconstruction of the mandible, a partial resection was performed in April 2001, and reconstruction bone plate was used to maintain the continuity of the mandible. After 3 months, a fracture of the bone plate was noted. Two bone plates were then used to provide stronger temporary reconstruction of the mandible. Bony reconstruction using a free fibular flap was performed in July 2003, and the patient made an uneventful recovery. The plates were removed in July 2004, and the patient remains stable enjoying satisfactory mandibular function and good cosmetic outcome (Fig. 1e). Distraction osteogenesis of the free fibular flap is planned before complete dental rehabilitation using osseointegrated dental implants.

\section{Case 2}

A 15-year-old boy was presented to our clinic in May 2003 with swelling in the right side of the mandible. The clinical examination revealed a local pulsation in the area of the caudal border of the body of the mandible. The panoramic radiograph revealed a vague radiolucency in the right body of the mandible ranging from the central incisor up to the second molar. MR examination showed a highly vascularised lesion in the right body of the mandible, and the differential diagnosis was AVM, angiosarcoma or telangiectatic osteosarcoma. In August 2003, an angiography demonstrated a high-flow AVM of the right mandible fed by the right mandibular, right and left facial and right maxillary artery (Fig. 2). In the same intervention, we carried out an arterial embolisation using Ethibloc (Ethicon, Johnson \& Johnson), and the post-embolisation control

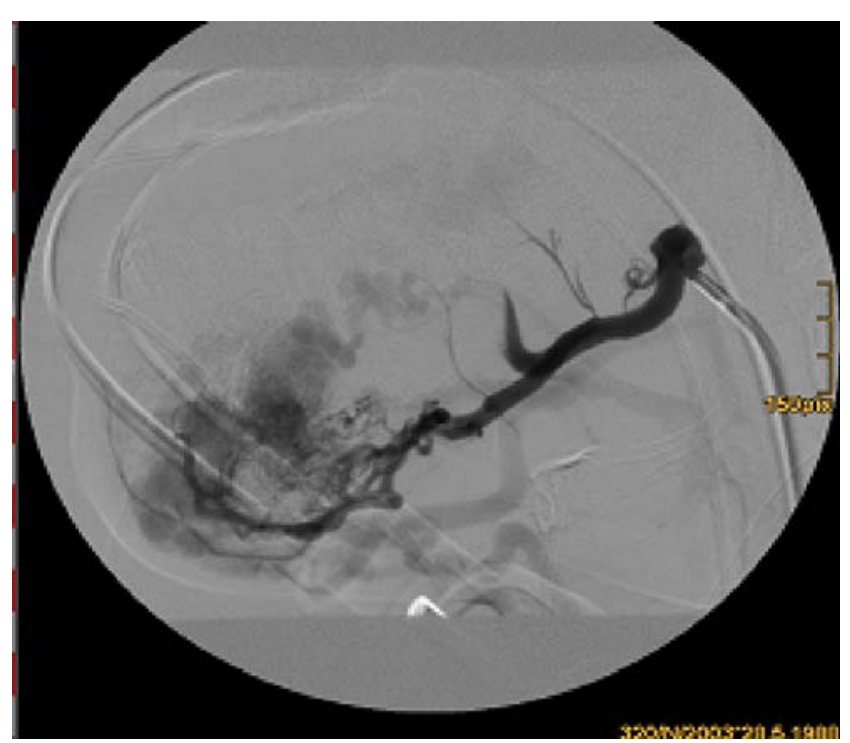

Fig. 2 Angiography demonstrating the AVM revealed a subtotal closure of the AVM. A repeat MR and angiography were planned for September 2003, but the patient did not keep the appointment.

Due to persistent swelling and light pain in the area of the right mandible, the patient visited our clinic again in November 2003. The angiography revealed a recurrence of the AVM; consequently, arterial embolisation of the right facial artery, left facial artery and right mandibular artery using Ethibloc (Ethicon, Johnson \& Johnson) was performed. The residual AVM was reduced but not completely eradicated, and the neuroradiologists suggested a subsequent surgical approach. The patient's mother did not agree to the operation. The repeat MR and angiography in January 2004 demonstrated a larger recurrence of the AVM, and a combined angiography-embolisation operation was strongly recommended as treatment, which was once again rejected.

Due to relapsing periodontal bleeding, the patient was hospitalised in May 2004. Arterial embolisation was followed 1 week later by intraosseous embolisation of the AVM with an application of butyl-2-cyanoacrylate (Histoacryl ${ }^{\circledR}$, Braun). Massive haemorrhage with pulsation occurred during the transosseous puncture of the lesion, despite the arterial embolisation. After obliteration of ten compartments of the AVM with cyanoacrylate, we achieved permanent closure of the AVM. A CT scan was performed 5 weeks after the intraosseous embolisation. A secondary resection was recommended, but the patient did not keep the appointment.

\section{Case 3}

An 11-year-old girl was referred to our clinic in February 2002 with the diagnosis of AVM of the left body of the mandible (Fig. 3). More than 50 interventions (arterial embolisation, coiling) under general anaesthesia had been performed up to then. None of these measures had brought about a permanent elimination of the AVM. In May 2002, a selective arterial embolisation of the AVM was performed in interdisciplinary cooperation with the Department of Neuroradiology. Permanent intraosseous embolisation of

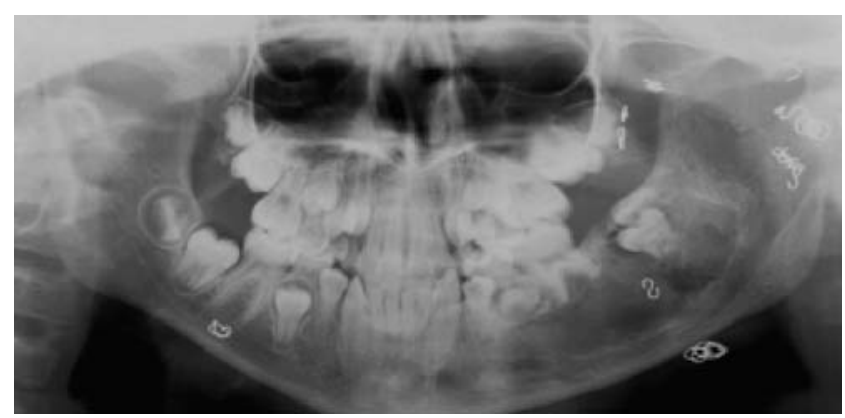

Fig. 3 Panoramic radiograph of the 11-year-old girl showing the AVM of the left mandible 
the cavities of the AVM with butyl-2-cyanoacrylate (Histoacryl ${ }^{\circledR}$, Braun) was undertaken, and the subsequent arteriogram showed no lateral or collateral perfusion and a permanent eradication of the AVM. In July 2002, partial resection of the mandible took place followed by alloplastic reconstruction by means of one reconstruction plate, and a bony reconstruction via iliac crest was performed in July 2003. The plates were removed in July 2004, and during the same operation, three dental implants were inserted in the augmented bone. The prosthetic rehabilitation was completed in July 2005.

\section{Discussion}

AVMs are extremely rare entities that could be lifethreatening if left untreated due to massive blood loss on the occasion of tooth extraction or biopsy [4-6]. Although rare, $50 \%$ of all intraosseous AVMs occur in the maxillofacial region [7] and extremely seldom in the mandible. These lesions can go unrecognised for years. However, symptoms commonly reported by patients with AVM are soft tissue swelling, pain of variable intensity, teeth mobility and migration, discoloration of overlying skin and intraoral mucosal surfaces, paraesthesia and facial asymmetry, local pulsation, audible bruit, palpable thrill, periodontal bleeding or spontaneous bleeding by tooth eruption or brushing teeth and bone resorption as well as resorption of the roots in the affected area without any evident periapical inflammatory procedure $[4,6,8]$. These symptoms are described in our cases.

The panoramic radiograph reveals no pathognomonic features of these lesions [6]. AVMs may have honeycomb radiolucencies or soap bubble appearance and can be interpreted as ameloblastomas, ameloblastic fibromas, odontogenic keratocysts, giant cell granulomas or malignant primary or metastatic tumors $[4,5,8]$.

A CT scan can show the shape, extent and boundaries of lytic expansion of intraosseous AVM, important before a direct intraosseous application of embolisation material [6]. Naturally, a superselective angiogram can provide crucial information on the feeder arteries, draining vein, flow rate and collateral flow of the AVM [6, 8]. These two examinations can be of great help for the definite diagnosis of an AVM.

Management of AVMs in the maxillofacial area is usually complex and requires multidisciplinary team approach. Regarding the treatment of the AVM, many options have been proposed. Curettage of the lesion can be curative, but may also lead to excessive bleeding [6]. Segmental resection or resection and a bone grafting procedure have also been proposed. Non-surgical treatment such as radiotherapy, injection of sclerosing agents and intravascular embolisation using polyvinyl alcohol foam, different kinds of coils and cyanoacrylate have been reported [9-11]. Serious complications after embolisation, such as occlusion of pulmonary or cerebral vessels with catastrophic consequences (e.g. paralysis, blindness or paralysis of the facial nerve) should also be considered $[12,13]$. Ligation of the external carotid artery should not be performed, firstly as many anastomoses promote the rapid appearance of a collateral circulation, and secondly because future embolisation would be impossible $[5,10]$. Other concepts include interventional radiology techniques, such as arterial embolisation combined with a surgical approach [5, 7]. Embolisation of feeding vessels may transiently reduce the size of the lesion as collateral vessels may develop within several days, as shown in our cases. Therefore, embolisation should be used in combination with intraosseous injection of embolisation agents to obliterate permanently the AVM [10]. In our first case, 1 year after the intraosseous embolisation, we had to treat a spontaneous fracture of the mandible, resect the necrotic mandibular bone and exposed cyanoacrylate and perform secondary reconstruction with a free fibular flap. Reossification of the lytic lesion described by Shultz et al. [14] and Flandroy and Pruvo [15] did not occur in our cases.

\section{Conclusion}

AVMs of the mandible may lead to fatal complications on the occasion of conservative dental treatment. The treatment of intraosseous AVMs requires a multidisciplinary team approach (anaesthesiologist, neuroradiologists, oral and maxillofacial surgeons). Extraosseous, superselective arterial embolisation is not always a permanent therapy. In cases of emergency, intraosseous application of an embolisation agent in the AVM, such as cyanoacrylate, can eliminate the danger of spontaneous, possibly fatal haemorrhage. To obtain stable results in the case of intraosseous arteriovenous malformations of the mandible, a combination of embolisation and surgery is recommended.

\section{References}

1. Higuera S, Gordley K, Metry DW, Stal S (2006) Management of hemangiomas and pediatric vascular malformations. J Craniofac Surg 17(4):783-789

2. Giaoui L, Princ G, Chiras J, Guilbert F, Bertrand JC (2003) Treatment of vascular malformations of the mandible: a description of 12 cases. Int J Oral Maxillofac Surg 32(2):132-136

3. Perugini M, Renzi G, Gasparini G, Cerulli G, Becelli R (2004) Intraosseous hemangioma of the maxillofacial district: clinical analysis and surgical treatment in 10 consecutive patients. J Craniofac Surg 15(6):980-985 
4. Mohammadi H, Said-al-Naief NA, Heffez LB (1997) Arteriovenous malformation of the mandible: report of a case with a note on the differential diagnosis. Oral Surg Oral Med Oral Pathol Oral Radiol Endo 84(3):286-289

5. Noreau G, Landry PP, Morais D (2001) Arteriovenous malformation of the mandible: review of literature and case history. J Can Dent Assoc 67(11):646-651

6. Fan X, Zhang Z, Zhang C et al (2002) Direct-puncture embolization of intraosseous arteriovenous malformation of jaws. J Oral Maxillofac Surg 60(8):890-896, discussion 896-897

7. Seehra J, Horner K, Coulthard P (2006) Arteriovenous malformation of the mandible - a case report. Br Dent J 201(1): 25-27

8. Engel JD, Supancic JS, Davis LF (1995) Arteriovenous malformation of the mandible: life-threatening complications during tooth extraction. J Am Dent Assoc 126(2):237-242

9. Akita S, Akino K, Tanaka K, Anraku K, Yano H, Hirano A (2006) Therapeutic choice for craniofacial venous malformations. J Craniofac Surg 17(4):729-735
10. Siu WW, Weill A, Gariepy JL, Moret J, Marotta T (2006) Arteriovenous malformation of the mandible: embolization and direct injection therapy. J Vasc Interv Radiol 12(9):1095-1098

11. Yoshiga K, Tanimoto K, Okui T, Kobayashi M (2003) High-flow arteriovenous malformation of the mandible: treatment and 7-year follow-up. Br J Oral Maxillofac Surg 41(5):348-350

12. Abouzgia MB, Symington JM (1992) Recurrent arteriovenous malformation of the mandible: a case report. J Oral Maxillofac Surg 50(11):1230-1233

13. Tang Chen YB, Wornom IL 3rd, Whitaker LA (1991) Intraosseous vascular malformations of the orbit. Plast Reconstr Surg 87(5):946-949

14. Shultz RE, Richardson DD, Kempf KK, Pevsner PH, George ED (1988) Treatment of a central arteriovenous malformation of the mandible with cyanoacrylate: a 4-year follow-up. Oral Surg Oral Med Oral Pathol 65(3):267-271

15. Flandroy P, Pruvo JP (1994) Treatment of mandibular arteriovenous malformations by direct transosseous puncture: report of two cases. Cardiovasc Intervent Radiol 17(4):222-225 